\title{
THE BIOFIDELIC BEHAVIOUR OF A CRANIO-CERVICAL SPINE SYSTEM IN SIDE IMPACT COLLISIONS
}

\author{
Minai Clinciu \& Stefanita CiUnel
}

Abstract: Even though the frontal collisions occur in a much larger percentage than the lateral collisions, the lateral collisions consequences can be much more serious than the frontal collisions. This paper is meant to be a study on the importance of crush energy which determines the mechanism who produces the severity of injuries. Using Newton laws of mechanics, we can discuss about the conservation of energy and linear momentum law which determine the impact force, speed and deformation. Therefore, in this paper the authors studied and analyzed the biomechanical behaviour of cranio (head) - neck (cervical spine) area of an anthropometric test devices for two different collision scenarios: the lateral collision between two vehicles and the collision between $a$ vehicle and a fixed rigid vertical cylindrical obstacle (tree, pole, e.g.). After, we compared the obtained data from the laboratory virtual crash tests with the determined values from the polygon experimental tests. Analytical techniques who determine the occupant and vehicle behaviour from this stage allow to determine the mechanism who produces the damages and biomedical parameters, which are representative and correspond too the real applications from the practice investigation of the traffic events. The information are extremely useful to expert investigators who activates in the field of the traffic events investigation and reconstruction.

Key words: vehicle, lateral collision, biomechanical, behaviour, cranio - cervical spine
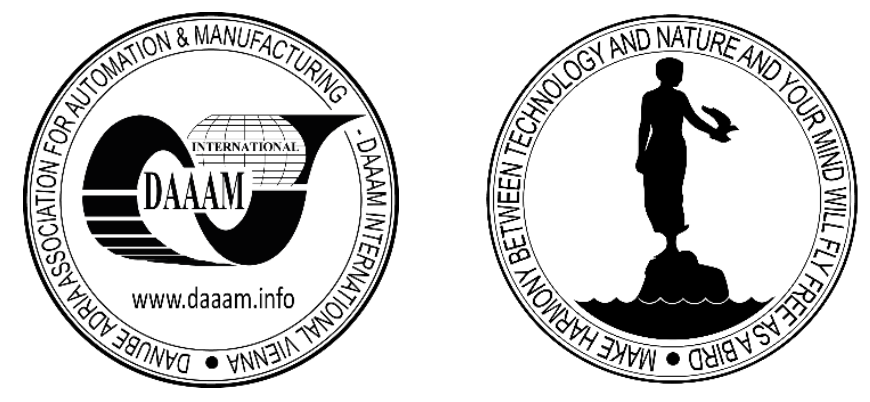

Authors' data: Clinciu, M[ihai]*; Ciunel, S[tefanita], * Transilvania University of Brasov, Faculty of Mechanical Engineering, Politehnicii Street No.1, Brasov, Romania, $500024 \quad$ Brasov, Romania, mihaiclinciu@yahoo.com, ciunel_stefanita@yahoo.com

This Publication has to be referred as: Clinciu, M[ihai] \& Ciunel, S[tefanita] (2019). The Biofidelic Behaviour of a Cranio-Cervical Spine System in Side Impact Collisions, Chapter 17 in DAAAM International Scientific Book 2019, pp.215-232, B. Katalinic (Ed.), Published by DAAAM International, ISBN 978-3-902734-24-2, ISSN 17269687, Vienna, Austria

DOI: $10.2507 /$ daaam.scibook.2019.17 
Clinciu, M. \& Ciunel, S.: The Biofidelic Behaviour of a Cranio-Cervical Spine Syst...

\section{Introduction}

The most common road events consist in frontal or lateral followed by rear end collision, respectively by rollover. Annually road events with side-impact car crashes generate victims whose mortallity accounts for $25 \%$ of their total. Load force who is acting in these type of collisions on the occupants are violent, because the space between the victim and the vehicle parts (knows as non-lethal space) is limited. After the collision, serious injuries result because the lateral structure of the vehicle is less rigid (stiffness) than the front $[2,10]$. Passenger safety is one of the biggest concerns of those involved in the automotive industry. Vehicle manufactures are required to carry out a variety of tests for each model that is on the market. Even though these tests can be easily evaluated, they are complex and complicated requiring appropriate facilities, data acquisition systems, qualified personnel, not to the mention the complete destruction of motor vehicles.

Because there is a long way to go from the design phase to the final prototype, for this reason, to prove if a vehicle meets the initial safety requirements, tests were carried out at different design phase, which were quite expensive. It is therefore advisable to establish a type of standard experimental test and to use large scale measurements to determine the behaviour of the vehicle and passengers during a collision. Methods for reconstructions of road events have evolved in line with the progress in related fields, such as modern methods of investigation and analyses of the scene accident, which have take topometry techniques, car maintenance, satellite surveillance, biomechanics, etc $[1,5,7]$.

In present, there are opportunity for aerial photography, digital camera shooting, distance measurement with laser equipments, examination of accident site with equipment than can give the value of adheresion coefficient, careful examination of vehicles involved in the road accident, careful indoor and outdoor photography, testing the effectiveness of the braking system on specialized stands, using the information provided by the electronic blocks, for monitoring brake functions, deploying airbag systems and pretension seat belts, to model the dynamics of the accident with computers.

The results obtained with these modern facilities have begun to overturn or fade the importance of witness statements that could appreciate the movement of the vehicles but could not appreciate the speed of their movements. Pictures are absolutely necessary for the evaluation of vehicle deformations and post-impact reciprocal positions. For the technical expert who cannot go to the road event, the images must allow a clear picture to be drawn on the scene accident, especially that the scene of the accident cannot be preserved for a long time. Reconstruction of road events is based on energy conservation dynamics, which directs the sequence of phases describing the road event.

Modeling and simulation of road events requires laborious technical procedures. In practice, engineers and technical investigators estimate the severity of the road event and the initial motion conditions with data such as braking distance, adherence coefficent and energy consumptions. 
Sometimes, the brake tracks are not preserved, taken or the vehicle moves on surface (wet, snow covered or heavey traffic areas) that do not retain traces of rubber. More models have been developed that allow technical investigators to correlate the damage geometry with the energy consumed in the collison and injury severity $[6,15]$.

Some of these analytical models put in evidence the correlation between the deformation and the speed of the vehicle before the collision. All these models aim to simulate the behaviour of the victim and the vehicle during the road event and the degree of accuracy is validated by comparing the data of interest offered by the simulation with the data obtained from the experimental laboratory tests or under real conditions.

\section{Evaluation of deformation energy}

An important objective of investigating road events is to determine the principal direction of force, the energy consumed in the collision at the deformation of the vehicle, the injury severity, respectively initial and final speeds. So, the kinetic energy during the road event, will be consumed by deforming the vehicle and by solicitation and injuring the car occupants. The size of specific load force which act on different parts of the human body is determining the factor who causing injuries and if the tolerance limits are exceeded.

Determinations that use the data regarding gravity of the damage assume to use of analytical models to express the correlation between the normal collision force on the unit of width of the damaged area and the amplitude of the deformations. Analytical models that take into account deformations can be static or dynamic Static models show the relationship between the force of collision and the residual plastic deformation of the vehicle, and the dynamic models put in evidence the relationship between the force of collision and the total deformation of the vehicle, assimilating this dependence as linear. The collision phase modeling will take into account the energy consumed at the deformation of the car body elements.

Because in the collision study, the energy consumed in deforming the car body, taking into account residual deformations is used to establish vehicle speed variation $\Delta \mathrm{v}$, the deformation of the vehicle will resemble with a helicoidally elastic element. Few information's and limited date about experimental crash tests require extrapolations to be analyzed. The linear dependence between the impact force and the size of the residual deformation allow to determine the stiffness coefficients. The most common method for establish deformation energy was introduced by Kenneth L. Campbell [9] and is also used in the Crash 3 software collision modeling algorithm $[11,18]$.

The models contain some simplifying assumptions such as: - residual deformations and stiffness coefficients are obtained from the experimental collision tests at impact speeds ranging from 45 to $55 \mathrm{~km} / \mathrm{h}$, preserving the force-deformation linearity hypothesis; - lost kinetic energy is transformed into mechanical work consumed in car structure deformation. The CRASH 3 program uses linear relationship between the vehicle structure resistance to deform and the residual deformation, which at first sight may seem like a simple empirical process. 
However, the only disadvantage would be that the collision data shows that this linear relationship fails to correlate with the results for extremely high speeds and deformations. However, despite this argument, the solution has been widely accepted throughout the scientific community. The energy determination in CRASH 3 program is based on the residual deformation of the vehicles involved in the collision. This is based on two hypotheses: the impact force is proportional to the residual deformation and the residual deformation is proportional to collision speed. First hypothesis is presented in Figure 1.

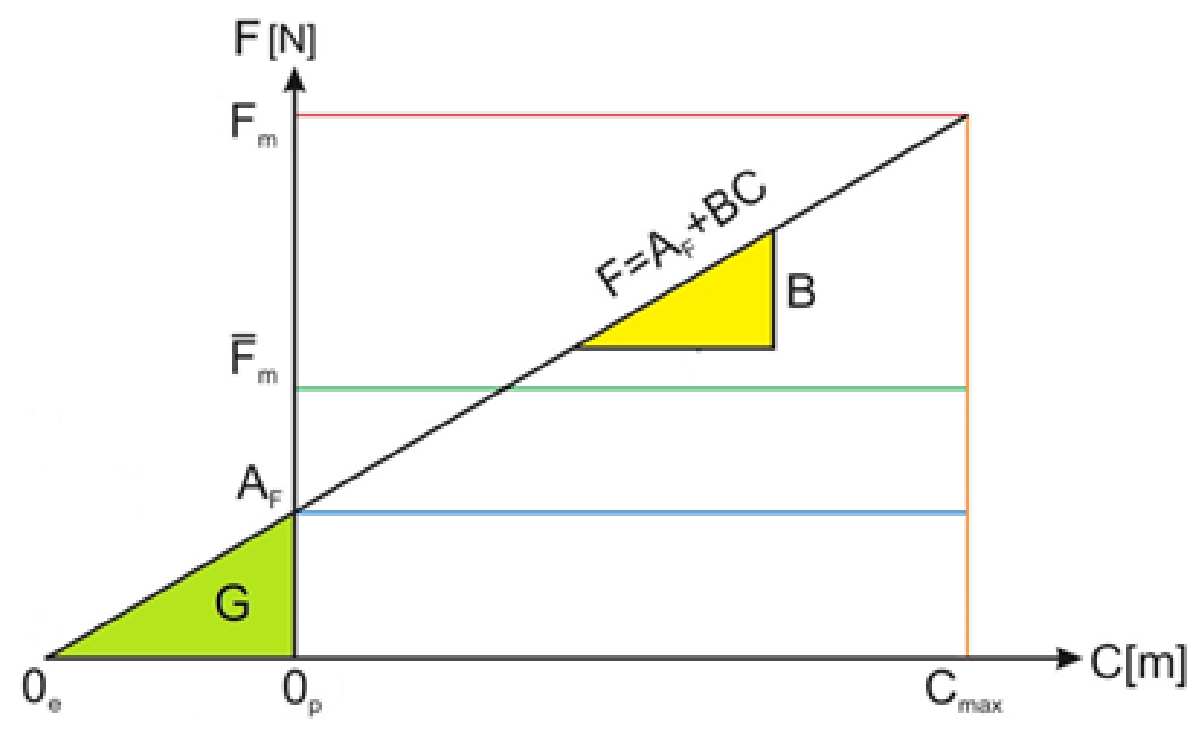

Fig. 1. Linear characteristic force - deformation

$$
F=A_{F}+B C
$$

where: $A_{F}$ is a stiffness coefficient $[N / m]$, which ensures that a force is taken without the contact area being deformed residually; $B$ - stiffness coefficient $\left[\mathrm{N} / \mathrm{m}^{2}\right]$, which represents the ratio of the maximum force over the unit width of the deformed area and the amplitude of the deformation and $C$ - the amplitude of the residual deformation $[m]$.

The energy needed for the deformation of the vehicle is:

$$
E=A_{F} C+\frac{B C^{2}}{2}+\frac{A_{F}^{2}}{2 B}
$$

The energy lost in the collision can also be expressed by the double integration of the relation (1), as follows:

$$
E=\int_{0}^{L_{z}} \int_{0}^{C}\left(A_{F}+B C\right) d c d l
$$




$$
E=\int_{0}^{L_{Z}}\left(A_{F} C+\frac{B C^{2}}{2}+\frac{A_{F}{ }^{2}}{2 B}\right) d l
$$

where $L_{z}$ is the width of the deformed profile.

The second hypothesis can also be presented graphically by a straight line Figure 2, but this time the ordinate is the impact speed rather than the impact force. Kenneth L. Campbell [9] notes that the impact speed and the amplitude of the residual deformations are proportional.

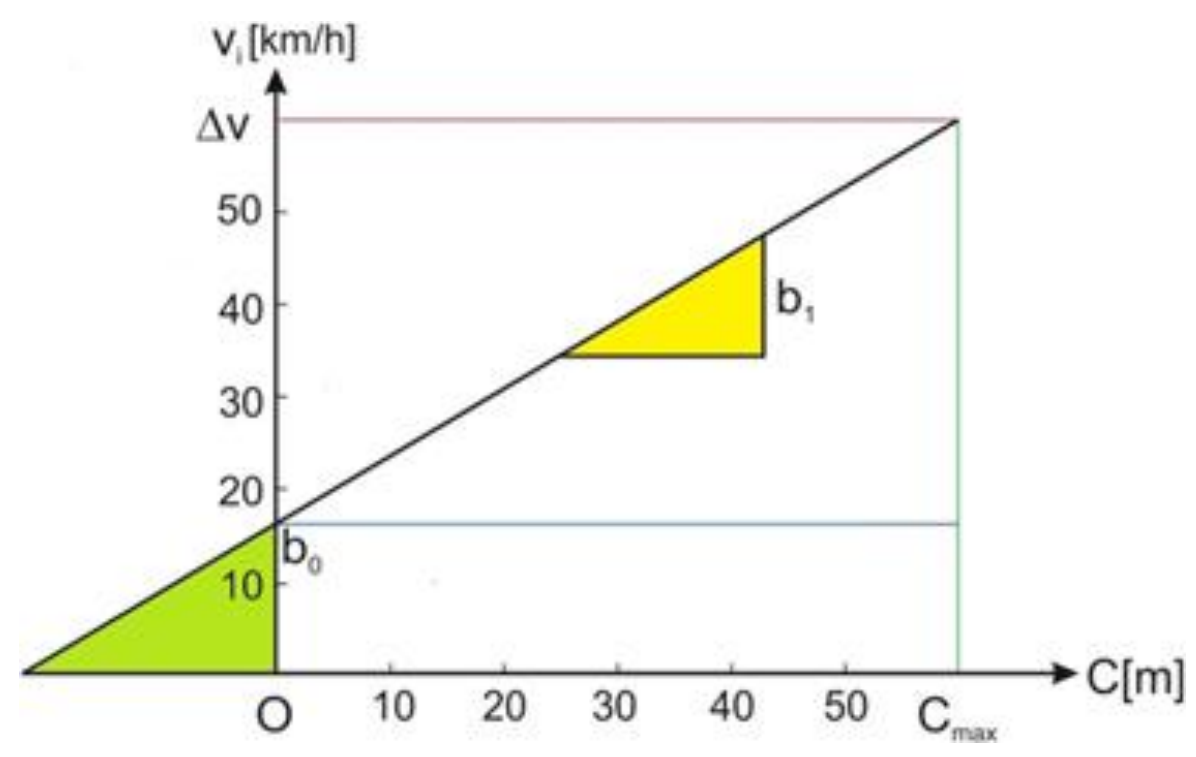

Fig. 2. Linear characteristic force - deformation

The dependence between the impact speed and the size of the deformation has a linear shape:

$$
v=b_{0}+b_{1} \cdot C
$$

where: $v$ - impact speed $[\mathrm{m} / \mathrm{s}] ; b_{0}$ - the maximum speed at which no residual deformation produce $[\mathrm{m} / \mathrm{s}]$;

$b_{1}$ - slope (ratio of the impact speed and the average amplitude of deformation) $[1 / s]$ and $C$ - the amplitude of the residual deformation $[\mathrm{m}]$.

These models allow simulation of the vehicle's behaviour respectively cars occupant behaviour during collision process, and the degree of accuracy is validated by comparing the data of interest obtained from the simulation process with those obtained experimentally under laboratory test or real conditions, showing the link between the amplitude of the deformation, injury severity and initial speed. 
Clinciu, M. \& Ciunel, S.: The Biofidelic Behaviour of a Cranio-Cervical Spine Syst...

The kinematic, dynamics and modeling analysis process of deformation are interdependent because the parameters of pre-crash phase conditioning the body deformation, which also influences the kinematic parameters of the post-crash phase. In the side impact collision the driver and occupants are subjected to high solicitation loads in comparison with other types of collision with the same level of crush energy absorbed [2,3].

During lateral collision with a vertical cylindrical obstacle of unique radius, the central forces induce to the vehicle a particular rotating angular velocity, Figure 3.

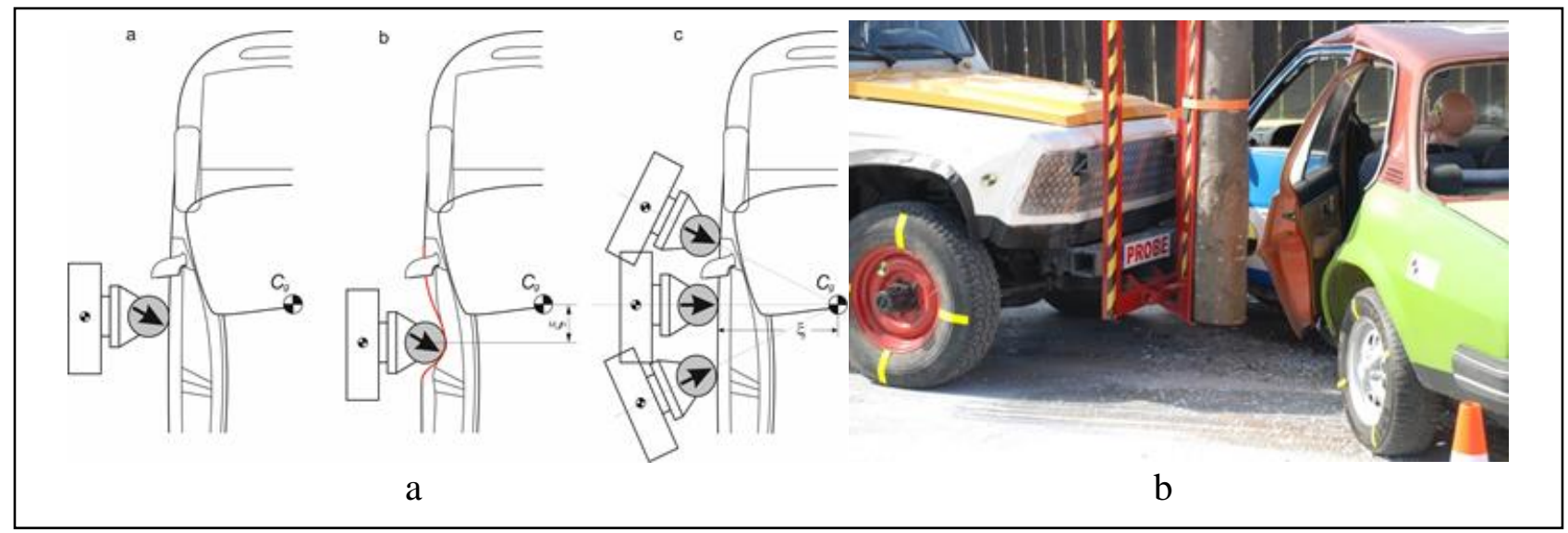

Fig. 3. (a) trajectory of collision, through center of mass; (b) real test

As a result, the vehicle will move in the direction of the resulting force, who acting on it, describing a rotation motion around its center of mass, Figure 4, in which:

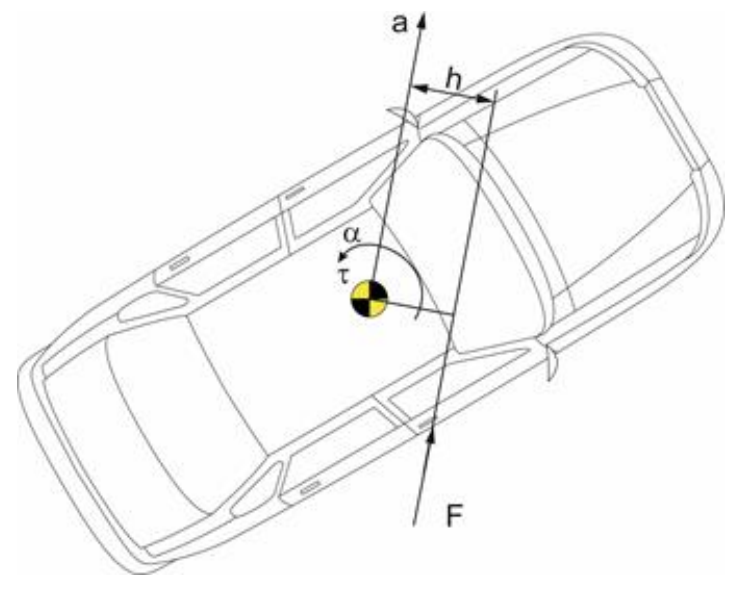

Fig. 4. Linear characteristic force - deformation

$F$ is the impact force $[N] ; a$ - mass center acceleration $\left[\mathrm{m} / \mathrm{s}^{2}\right]$, which overlaps with the direction of the resulting force of collision; $h$ - normal distance to the trajectory of the collision force $[\mathrm{m}] ; \tau$ - the moment of gyration [Nm], being the product of $F$ și $h$; $\alpha$ - angular acceleration of gyration $\left[\mathrm{rad} / \mathrm{s}^{2}\right]$. 
This induced angular acceleration will continue to grow, with the deformation of the vehicle body elements, until it reaches the maximum level. For calculating the energy absorbed by deformation of the vehicle structure resulting rotational energy must be subtracted from the total energy of the impact.

The rotation induced as a result of vehicle impact is composed of:

- rotating phase of the vehicle around the obstacle collided;

- rotation phase of the vehicle after separation from the obstacle collided.

At this stage, the rotational speed decreases thanks to interaction between tire and road surface area. In general the angular velocity reaches the maximum value at the end of the collision phase.

For the movement of gyration of Newton's second law, we have:

$$
\tau=I_{\psi} \alpha
$$

$I_{\Psi}=$ moment of inertia $=m r_{g}^{2}, r_{g}^{2}=\frac{I_{\Psi}}{m}$

for motor vehicles the moment of inertia is: $I_{\Psi}=3,079 \cdot m-1635$

with $r_{g}$ - radius of gyration;

from which it result:

$$
\tau=m r_{g}^{2} \alpha
$$

or the moment can be written as follows:

$$
\tau=F h
$$

If

$$
\begin{aligned}
& a_{c}=a+h \alpha \\
& \alpha=\frac{a_{c}-a}{h}
\end{aligned}
$$

Then

$$
\begin{aligned}
& F h=m r_{g}^{2} \alpha \\
& F h=m r_{g}^{2}\left(\frac{a_{c}-a}{h}\right) \\
& \frac{F}{m} h^{2}=r_{g}^{2}\left(a_{c}-a\right)
\end{aligned}
$$


Clinciu, M. \& Ciunel, S.: The Biofidelic Behaviour of a Cranio-Cervical Spine Syst... knowing that: $F=m a \quad$ or $\quad a=\frac{F}{m}, \quad$ result:

$$
\begin{aligned}
& a h^{2}=r_{g}^{2}\left(a_{c}-a\right) \\
& a=\left(\frac{r_{g}^{2}}{r_{g}^{2}+h^{2}}\right) a_{c}
\end{aligned}
$$

We will define the dynamic ratio as being $\gamma=\frac{r_{g}{ }^{2}}{r_{g}{ }^{2}+h^{2}}$. Substituting $\frac{r_{g}{ }^{2}}{r_{g}{ }^{2}+h^{2}}$ with $\gamma$ in (13) is obtained the mass center acceleration of the vehicle, which is equal to the proportion $\gamma$ from the acceleration of the components located in the deformed zone:

$$
a=\gamma a_{c}
$$

The dynamic ratio will always be subunit or at most equal to 1 in case of a centric collision without gyration. To correlate the dynamic ratio $\gamma$ with change in velocity $\Delta v$ we can say that: $\Delta v=a \cdot \Delta t$ or $a=\frac{\Delta v}{\Delta t}$, and implicit: $a_{c}=\frac{\Delta v_{c}}{\Delta t}$.

so, from (14) result:

$$
\begin{aligned}
& \frac{\Delta v}{\Delta t}=\gamma \frac{\Delta v_{c}}{\Delta t} \\
& \Delta v=\gamma \Delta v_{c}
\end{aligned}
$$

In noncentric collisions, the common acceleration $a_{c}$ and change in velocity $\Delta v$ of the deformed volume elements will show values higher than those of the mass center. It is known that at centric collisions, the direction of the resulting collision force passes through the mass center of the vehicle and through the mass center of the deformed volume, which allows its determination of $\Delta v$, therefore the mass center of the deformed volume and the mass center of the vehicle will have the same change in velocity.

$$
\begin{aligned}
& \Delta v=\sqrt{\frac{2 E}{m_{1}\left(1+\frac{m_{1}}{m_{2}}\right)}} \\
& \Delta v_{c}=\sqrt{\frac{2 E}{\gamma m_{1}\left[1+\frac{\gamma m_{1}}{\gamma m_{2}}\right]}}
\end{aligned}
$$




$$
\Delta v=\gamma \sqrt{\frac{2 E}{\gamma m_{1}\left[1+\frac{\gamma_{1} m_{1}}{\gamma_{2} m_{2}}\right]}}=\sqrt{\gamma^{2} \frac{2 E}{\gamma m_{1}\left[1+\frac{\gamma_{1} m_{1}}{\gamma_{2} m_{2}}\right]}}=\sqrt{\frac{2 \gamma E}{m_{1}\left(1+\frac{\gamma m_{1}}{\gamma m_{2}}\right)}}
$$

\section{Virtual model and morphology of the neck-head system}

To obtain the virtual components of the human cervical spine [8] were analyzed 351 $\mathrm{CT}$ images obtained in different planes (distance between planes is $1 \mathrm{~mm}$ ), Figure 5 .

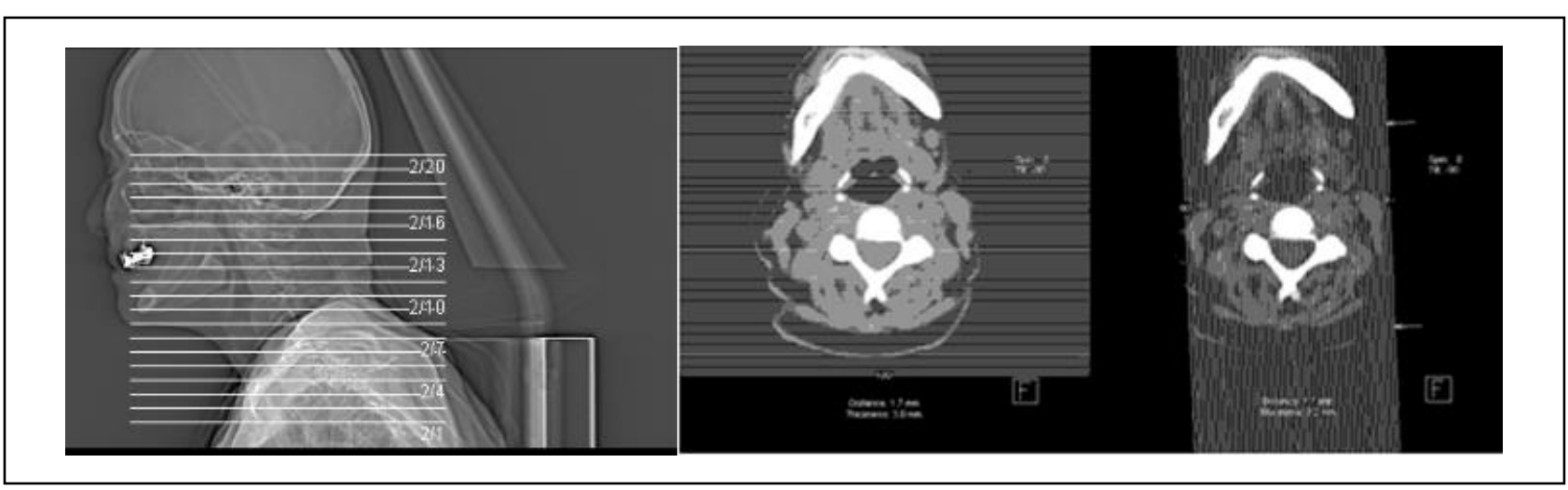

Fig. 5. Schemes used in tomography scanning cranio-cervical area

These images were scanned to a natural scale (1:1) and transfer, one by one, in a parametrical CAD environment where the contours became curves. After that operation the curves were unified in solids using Loft shape and the cervical spine were recomposed in a virtual space. In Figure 6 were presented the virtual model of the C1 (Atlas) and the entire virtual cervical spine composed by seven vertebrae.

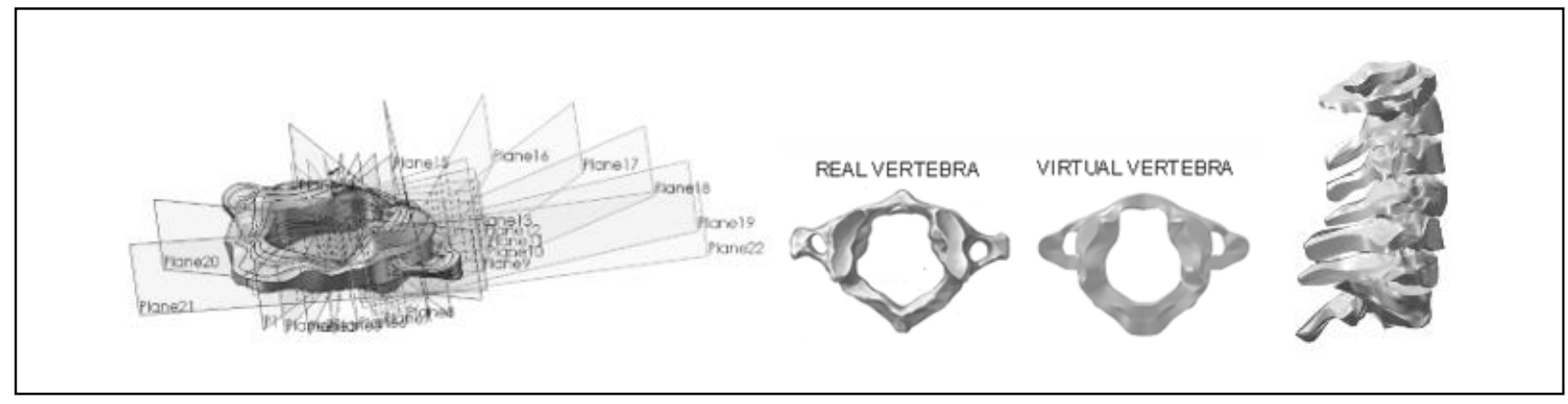

Fig. 6. Virtual morphology of atlas and cervical spine

Using the methods and commands exposed above and tomography images were obtained the models of the skull and upper torso bones. Using information from anatomical literature data, these virtual components were made and assembled with cervical spine previously defined [13]. In Figure 7 is shown the complete assembly of head-neck (cervical spine) biomechanical system in general views and details, for simulation using elastic elements which simulate the complex cranial-cervical muscle activity 

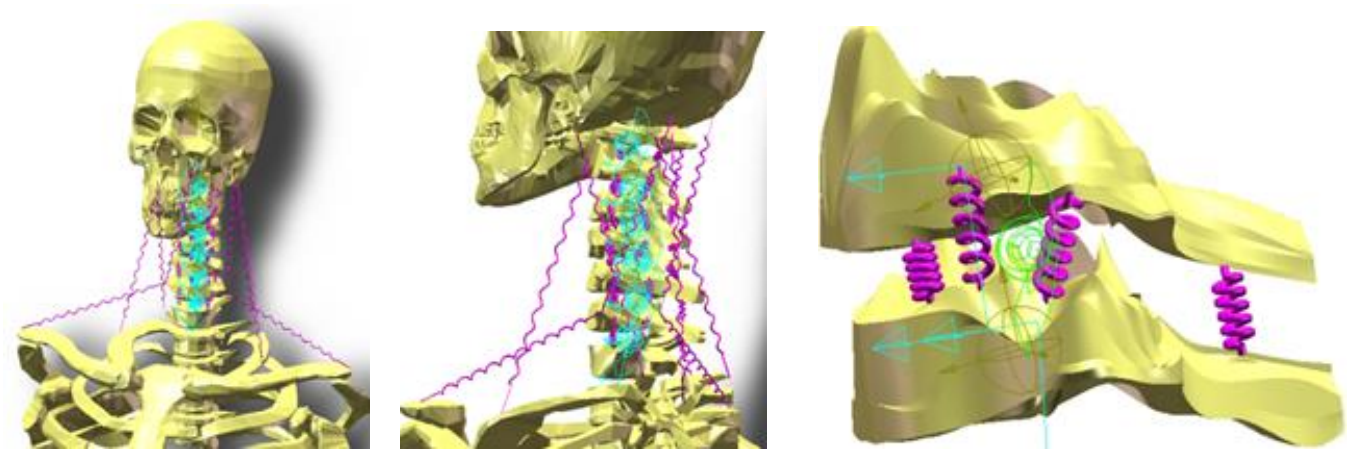

Fig. 7. The complete morphology of the virtual biomechanical system for the human head-neck (cervical spine) simulation actuated by virtual muscles.

A first result obtained is the film simulation. Figure 8 presents four important frames of the simulation, using driver and driven elastic elements as virtual muscles.

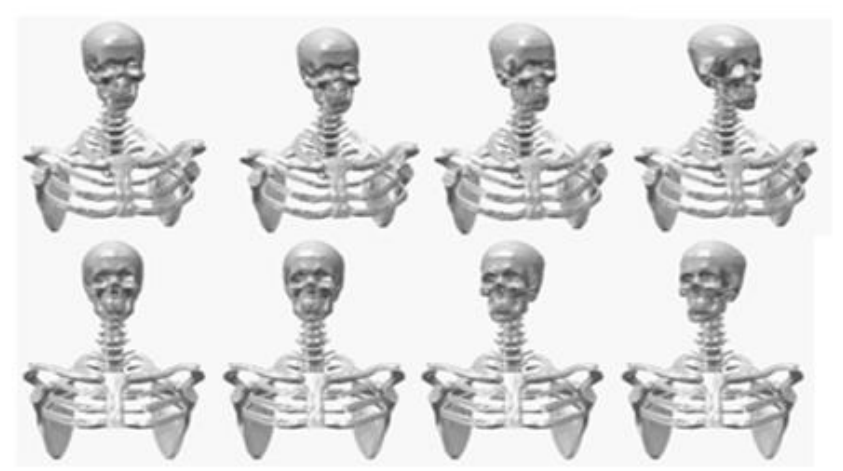

Fig. 8. Four major frames of the simulation movie (left and right axial rotation)

It also can be extract important kinematics parameters of any component of the studied biomechanical system. In Figure 9 were presented a few important parameters obtained automatically from the biomechanical system simulation for $\mathrm{C} 1-\mathrm{C} 2$ joint.
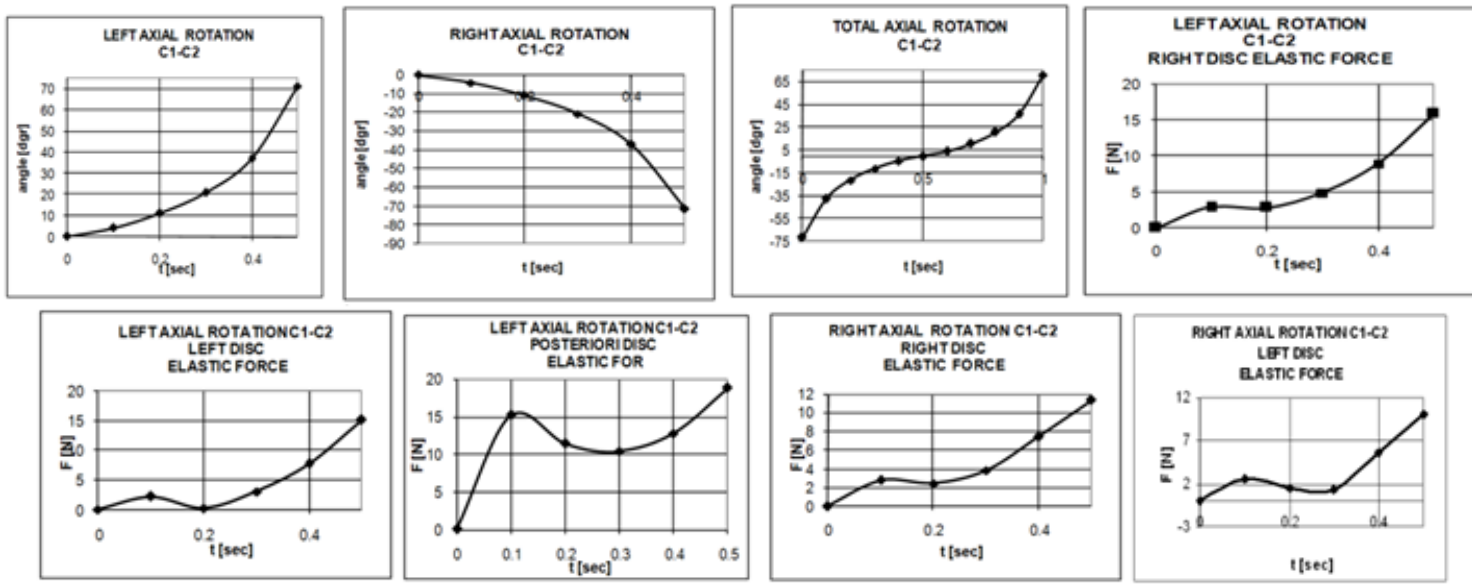

Fig. 9. Important kinematics parameters obtained from biomechanical analysis simulation system 
In a similar way, other parameters can be obtained for the other couplings of the human neck (C2-C3, C3-C4, C5-C6, C6-C7). Using the same model, but with other driver elements (other virtual actuated muscles) we obtained simulations for lateral bending and flexion-extension movements, Figure 10, with active elastic elements (similar studies in $[12,14]$ ).

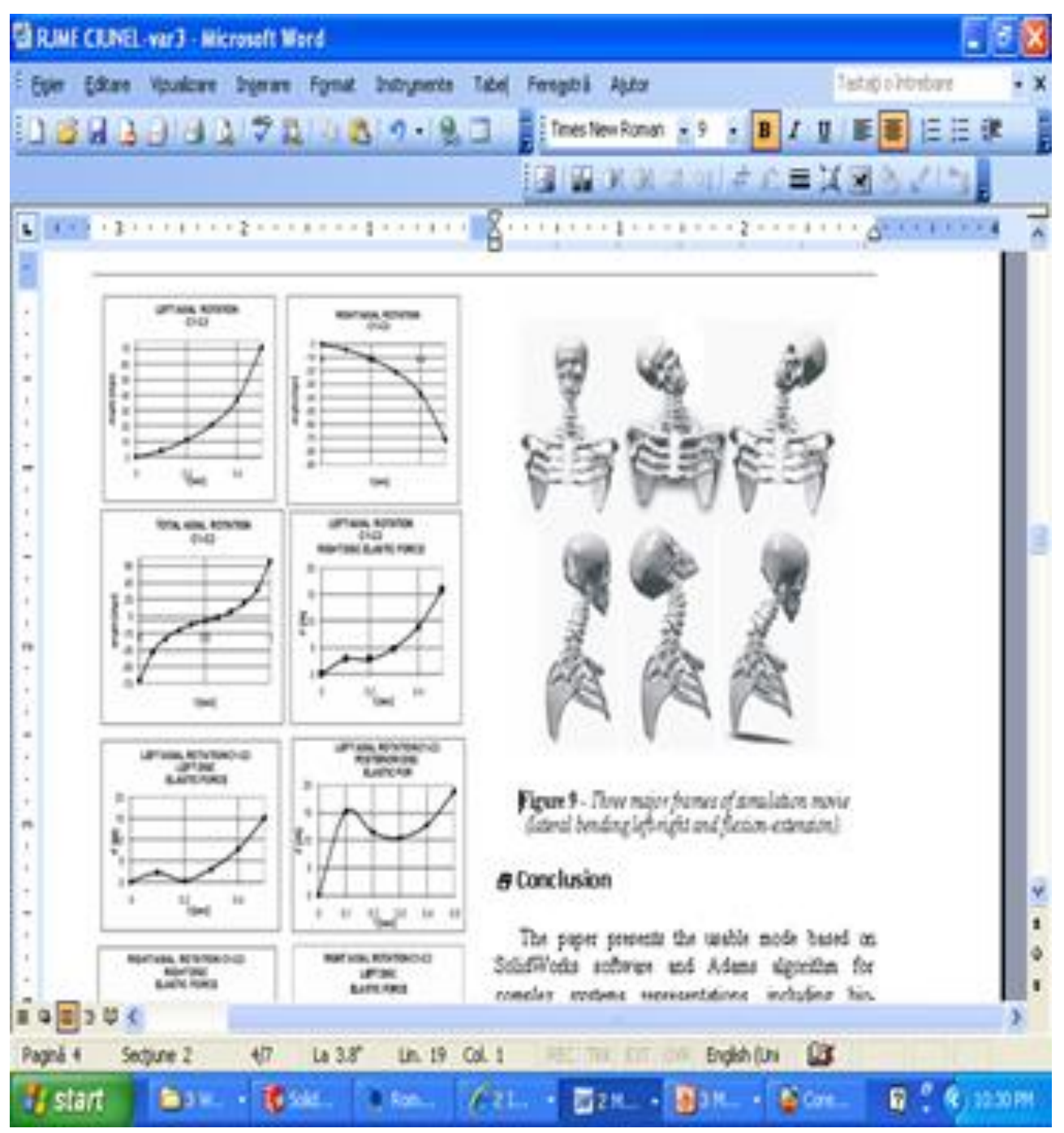

Fig. 10. Three major frames of simulation movie (lateral bending left-right and flexionextension)

\section{The Kinematic Simulations for the Main Movements of the Cervical-Head System}

After the modeling operation were extracted mass inertial and geometrical parameters for the components of the cervical spine. Some of them are calculated relative to the global coordinate system of the virtual model and any constants were taken from the literature. In Table 1 were presented the main inertial and geometrical parameters of the $\mathrm{C} 1-\mathrm{C} 7$ vertebrae. 
Clinciu, M. \& Ciunel, S.: The Biofidelic Behaviour of a Cranio-Cervical Spine Syst...

\begin{tabular}{|c|c|c|c|c|c|c|c|}
\hline & $\mathrm{C} 1$ & $\mathrm{C} 2$ & $\mathrm{C} 3$ & $\mathrm{C} 4$ & $\mathrm{C} 5$ & C6 & $\mathrm{C} 7$ \\
\hline Mass (g) & 170.62 & 153.20 & 172.79 & 174.75 & 222.38 & 237.59 & 289.57 \\
\hline Volume $\left(\mathrm{mm}^{3}\right)$ & 12274.7 & 11021.56 & 12430.7 & 12571.7 & 15998.35 & 17093.12 & 20832.37 \\
\hline Area $\left(\mathrm{mm}^{2}\right)$ & 5866.08 & 5689.36 & 5611.71 & 5571.39 & 6358.19 & 6617.17 & 7105.7 \\
\hline $\begin{array}{l}\text { Average } \\
\text { density } \\
\left(\mathrm{g} / \mathrm{mm}^{3}\right)\end{array}$ & \multicolumn{7}{|c|}{0.0139} \\
\hline $\begin{array}{c}\text { The modulus } \\
\text { of elasticity E } \\
\left(\mathrm{N} / \mathrm{mm}^{2}\right)\end{array}$ & \multicolumn{7}{|c|}{11400} \\
\hline $\begin{array}{c}\text { Poisson } \\
\text { coefficient }\end{array}$ & \multicolumn{7}{|c|}{0.39} \\
\hline
\end{tabular}

Tab. 1. The main inertial and geometrical parameters of the C1-C7 vertebrae

Study on biomechanical system was made in terms of simplifying assumptions: - the bones of the shoulder and upper chest were considered fixed (ground parts); - C1-C7 vertebrae were considered mobile (moving parts); - intra-vertebral articulations were considered spherical joints - 3R (Figure 7); - contact between the vertebrae was considered of "impact with friction" to eliminate virtual interference between solids; inter-vertebral discs between the vertebrae were simulated using four compression virtual springs except C1-C2 joint where were used three virtual springs (Figure 4); - virtual springs simulate, on the one hand, inter-vertebral discs with spring constant $\mathrm{k}$ $=2 \mathrm{~N} / \mathrm{mm}$ and for the springs located on the left or right side the spring constant is $\mathrm{k}=$ $20 \mathrm{~N} / \mathrm{mm}$ for the springs located front or rear and on the other hand the spring simulate the muscle action.

Also, the springs become actuators for the left and right lateral bending, and anterior and posterior actuators for extension and flexion. The biomechanical system of the head-cervical spine complex was simulates using initial action parameters and functions extracted from literature [17]. For example, in Figure 11 were presented twelve important frames of the lateral bending in side collision simulation and in Figure 12 and Figure 13 was shown the velocity and acceleration diagram for C1-C6 vertebrae during side collision simulation.

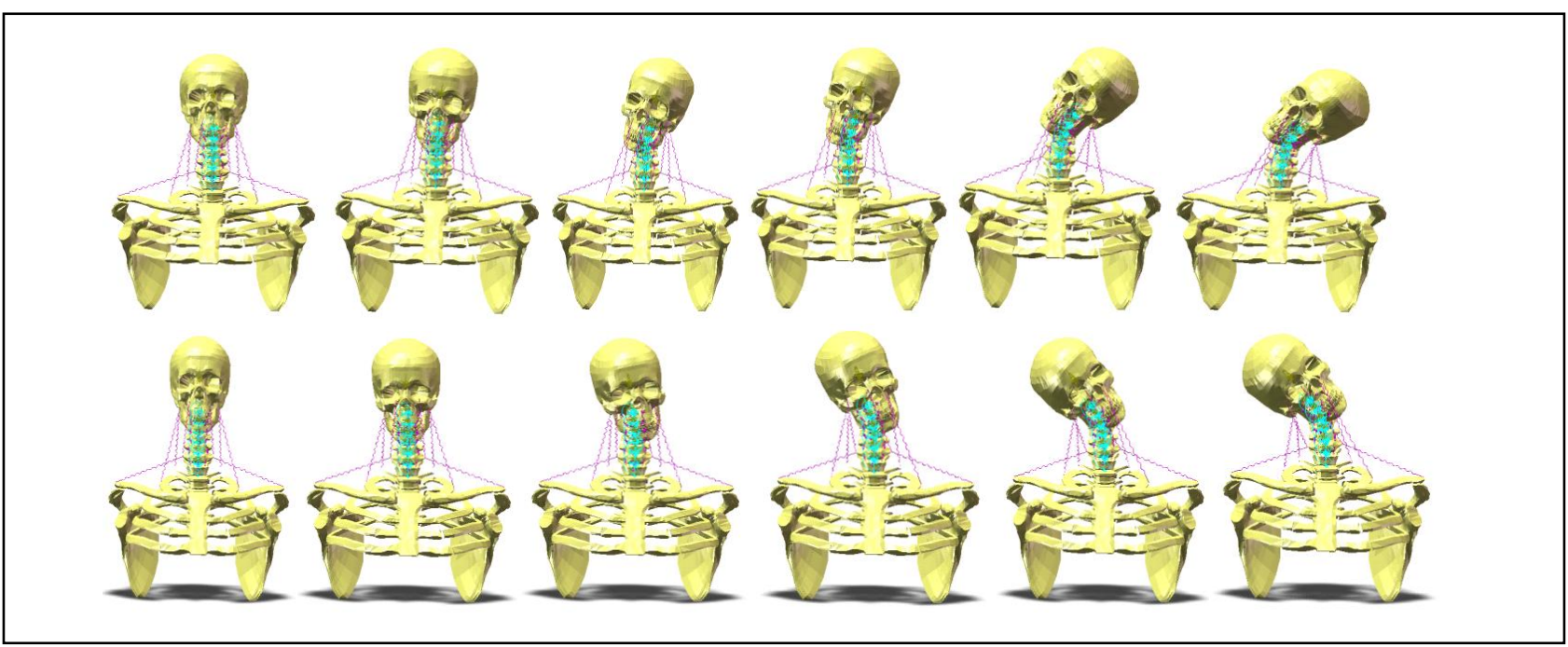

Fig. 11. Lateral bending simulation 


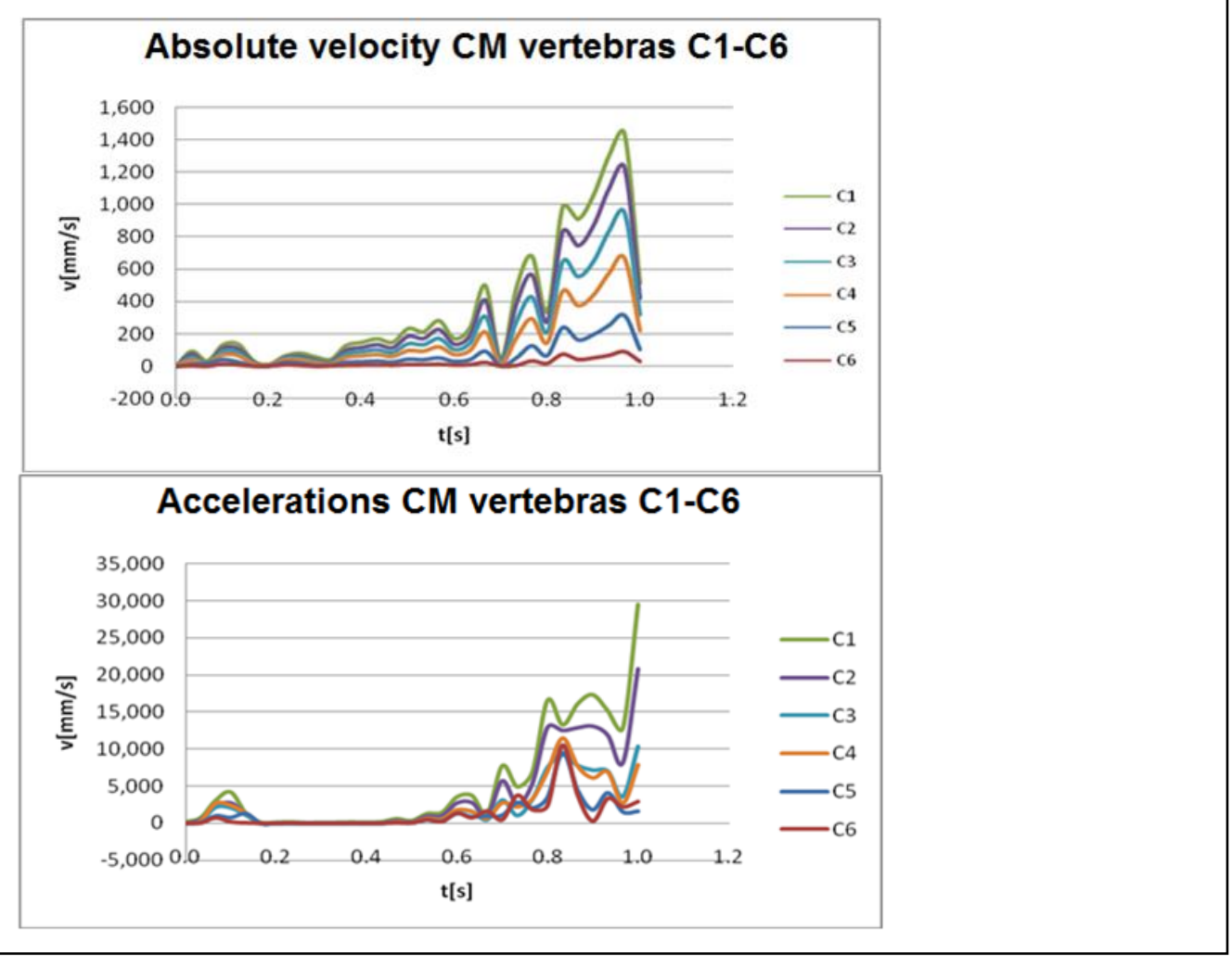

Fig. 12. Velocity and accelerations on left side

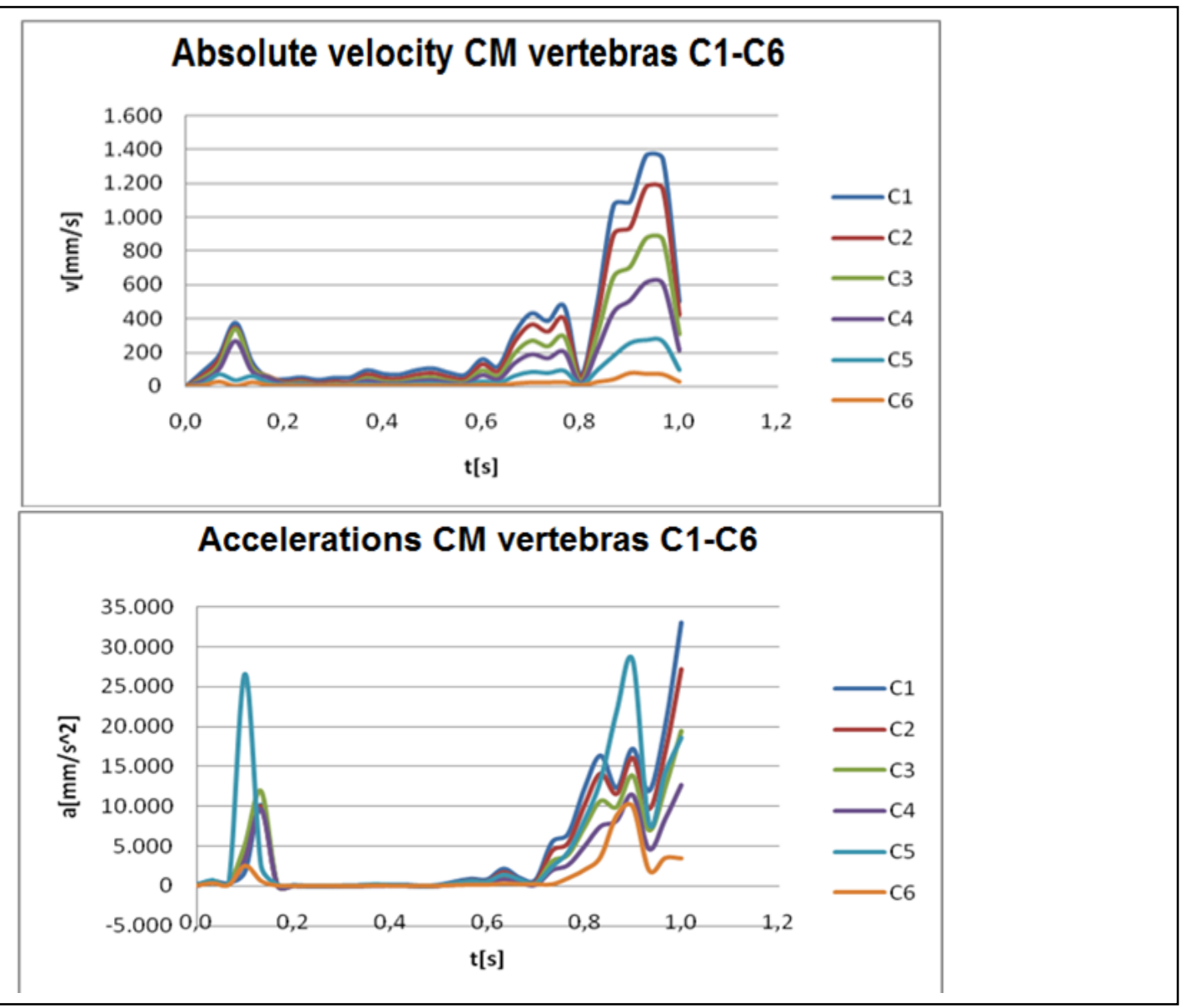

Fig. 13. Velocity and accelerations on left side 
Clinciu, M. \& Ciunel, S.: The Biofidelic Behaviour of a Cranio-Cervical Spine Syst...

Similarly, after the lateral collision simulation, have been obtained the evolution in time of forces and relative angles in intervertebral springs for $\mathrm{C} 1-\mathrm{C} 7$ vertebrae, Figure 14 and Figure 15.

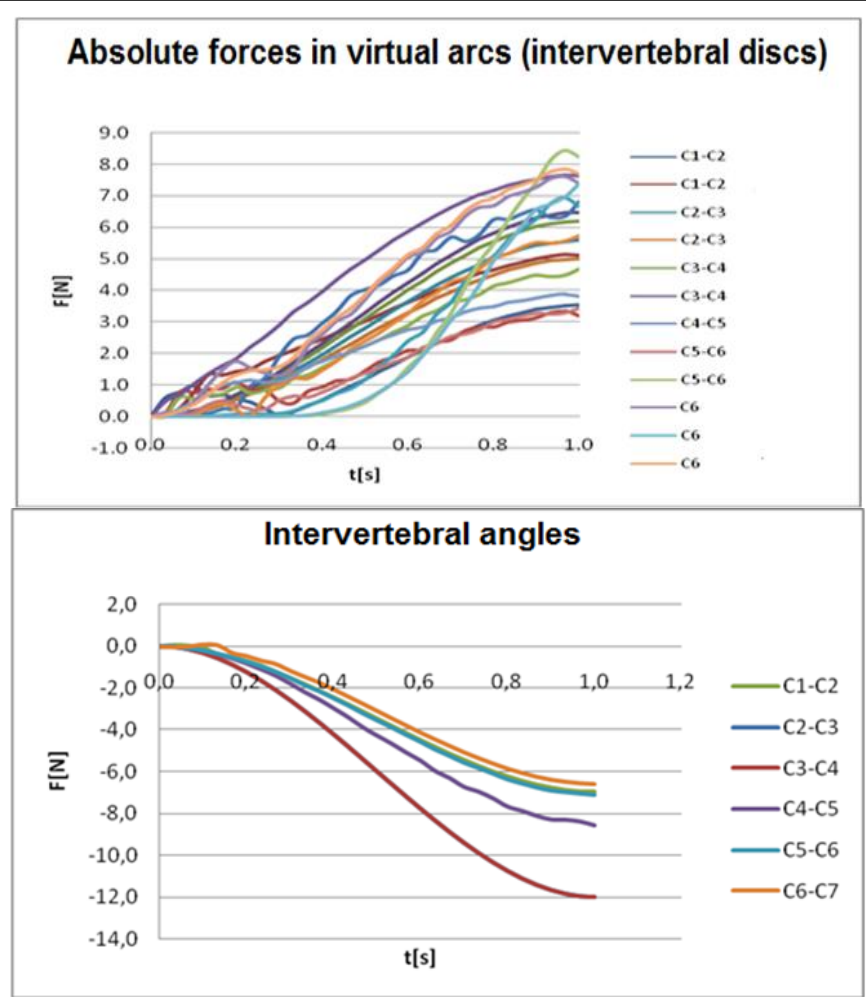

Fig. 14. Arc forces and intervertebral angles on left side

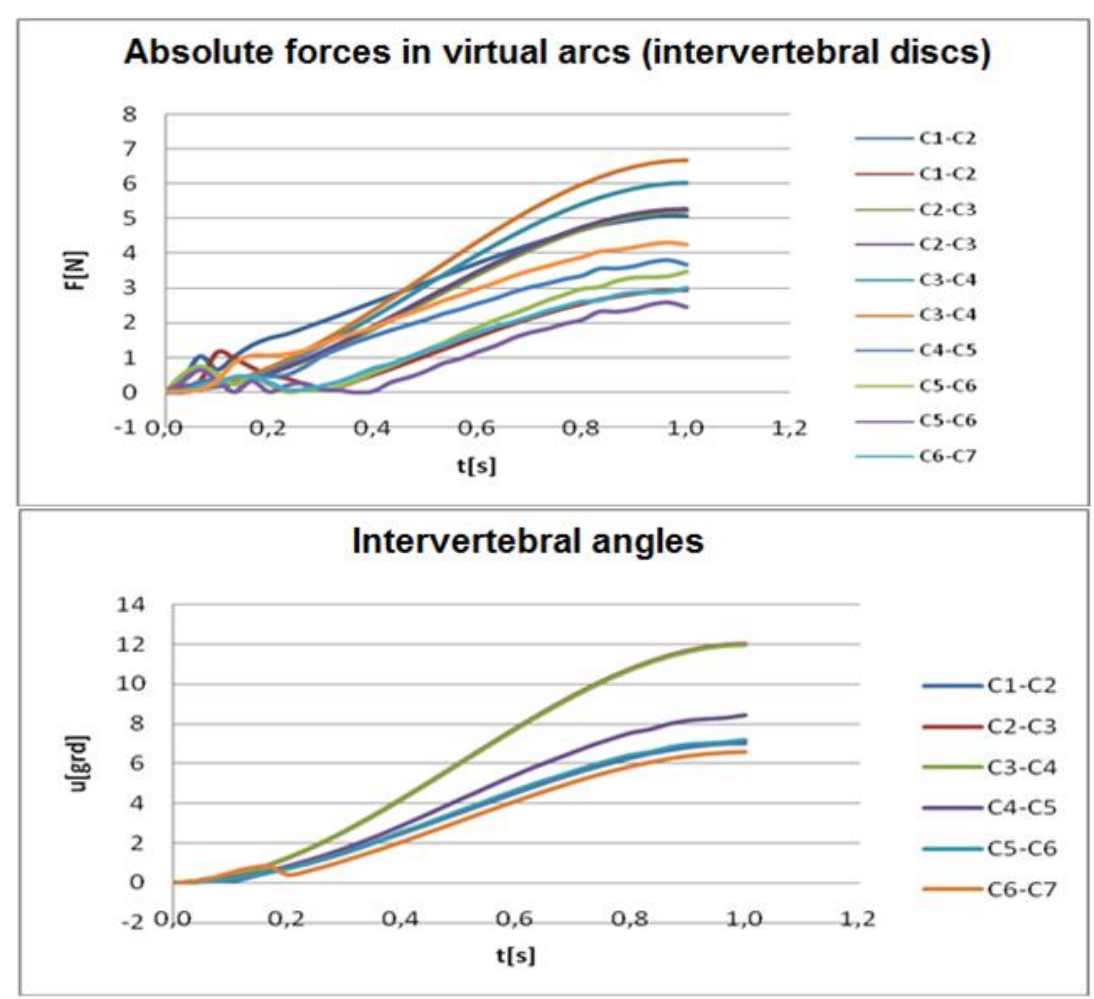

Fig. 15. Arc forces and intervertebral angles on right side 


\section{Conclusion}

The paper presents the studies made on a human similar biomechanical system composed by neck, head and thorax bones. The models were defined in a CAD environment which includes Adams algorithm for complex systems representations, including bio-mechanics, and the usable mode based on SolidWorks software for dynamic simulations $[17,19]$. The presented method is easy and simple, is working step by step and it offers the possibility to understand all the phases of the simulation operation. This software offers the complete access to the usable shape types and to the geometric and mass parameters. The paper shows the advantages of this CAD software, which become a high performance instrument for any complex bio-mechanical system, modelling and analysis, method used in [16]. The virtual models were obtained starting with tomography images made on a living human subject. The main movements analyzed were: lateral bending (left-right) and flexion-extension movement. After simulation was obtained the entire mechanical behavior based on data tables or diagrams. That virtual model composed by neck and head can be included in complex system (as an automotive system) and supposed to impact simulations (virtual crash tests). Based on simulation results will be developed an experimental testing system based on SMA (Shape Memory Alloys) springs used as artificial muscles. This system will be included in an experimental model for impact testing, Figure 16. The components have similar mass and inertial parameters as the virtual system analyzed by kinematic simulations presented in this paper.

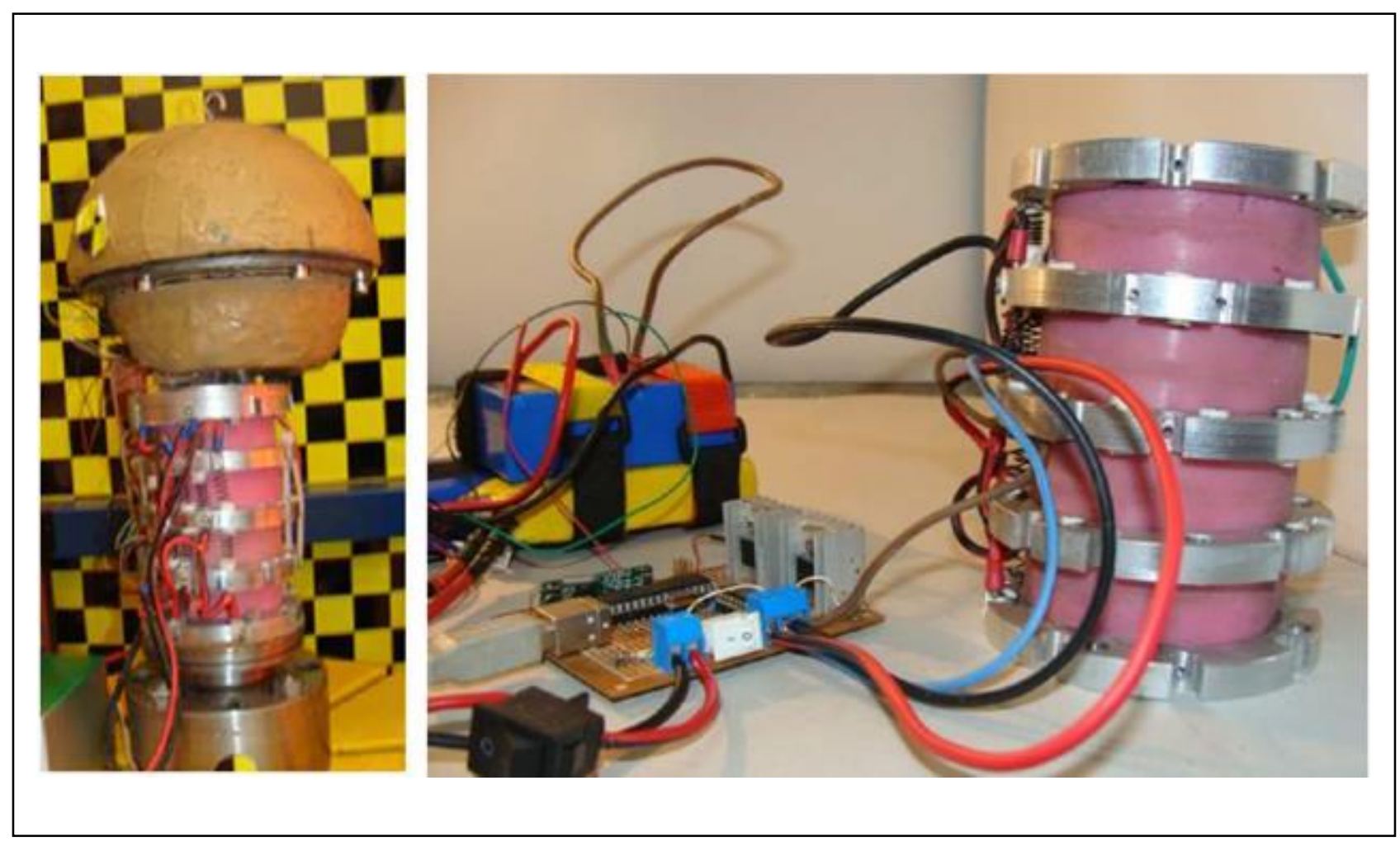

Fig. 16. Skull-cervical spine system actuated by artificial muscles of SMA springs under construction a) main skull-neck assembly, b) artificial cervical spine system with command and control systems 
Clinciu, M. \& Ciunel, S.: The Biofidelic Behaviour of a Cranio-Cervical Spine Syst...

That method for modelling and analysis has a lot of advantages as:

- the easiest way for understanding the elements and the components of the biomechanical system;

- simple and rapidly way to obtain an entire system of results using FEA (Finite Elements Analysis) calculus;

- using the module for FEA and kinematic simulation the virtual model can give important results as dynamic maps, data tables and graphical representations;

- a complex bio-mechanical system, like neck-head model, can be easily adapted to the new constrains and new parameters of the virtual crash test $[4,10]$.

- also, in our study were investigated similar devices which are components of other mechanisms from modern vehicles [14].

As well the results of this study allow generalization of the characteristics of energy dissipation in the vehicle lateral side. During collision of a fixed vertical cylindrical obstacles the energy turns in crush energy of the vehicle side, and the information regarding the deformation can be used in reconstructing road events. Only data obtained from tests vehicle collisions are compared with similar technical characteristics.

The procedures for investigating road events suppose that to determined the crush energy with the measured deformations of the vehicle it help in determining the vehicle speed at that moment. So the quantity of deformation energy for vehicle can be checked by providing energy losses with the help of dynamic timing analysis (CRASH III, PC- Crash, Virtual- Crash). Using hand calculation methods, we can obtain reasonable estimations, instead they are extensive, laborious and requires deep knowledge in the field [9].

The paper presented a test procedure where is performed a reversibly collision, namely the obstacle collides the vehicle which is stationary. Although this working mode is not always met in practice, the test procedure may be considered valid to study the energy absorbed during road event. Modern vehicles must meet consumer requirements regarding performance and safety features. One should remember that the results of experimental tests could be erroneously interpreted and conclusion could be erroneously placed that the lateral limits of the cabin are not capable to provide nonlethal space to the passengers. Test procedures used in the present are expensive and requires time in relation to the proposed solution.

The proposed solutions come to support road events research and implicitly determine the causes of their occurrence. In the future this experimental test procedure is intended to be extended to the rear side and rollover occupant behavior during road events research. 


\section{Acknowledgements}

This work was partially supported by the grant number 46C/27.01.2014, awarded in the internal grant competition of the University of Craiova, Romania. We thank all those who have financially supported the experimental research of the contract.

\section{References}

Brach R. M., Brach R. M. Vehicle Accident Analysis and Reconstruction Methods, Pennsylnvania, USA: SAE International, 1998.

Brach R. M. Comments on Energy loss in vehicle to vehicle impact by Dario Vangi. (2009) International Journal of Impact Engineering. May 2009.

Brach R. M. Crush Energy and Planar Impact Mechanics for Accident Reconstruction. SAE Paper 2011.

Bourdet N., Willinger R., Coupled head-neck-torso and seat model for car seat optimization under rear-end impact, Journal of Sound and Vibration 313, Elsevier, pp. 891-907, 2008.

Burg H., Moser A. Handbook of Accident Reconstruction, Kindle Edition, 2013.

Feng, Ch., Suren, Ch.: Injury severities of truck drivers in single and multi vehicle accidents on rural highways, In Accident Analysis \& Prevention, September 2011, Volume 43 issue 5, pp. 1677-1688, Published by ELSEVIER BV, 2011, USA, doi:10.1016/j.aap.2011.03.026.

Franch, H., Franch, D. Mathematical Methods for Accident Reconstruction. CRC Press, 2010.

Keller M., Development of a Cervical Spine Model for Rear Impact Conditions, Undergraduate Honors Thesis, Undergraduate Program in Mechanical Engineering, The Ohio State University, 2010.

K. L. Campbell, "Energy basis for collision severity", SAE Technical Paper Series, no. 740565,1974 .

Maltese M.R, et al, Injury Mechanisms in Seat Belt-restrained Occupants in Side Impact Crashes, Crash Injury Research and Engineering, Network (CIREN) Public Meeting Washington, DC, USA, March 28, 2007

McHenry Software, CRASH3 Technical Manual.

Netter F. H., Atlas of Human Anatomy, Icon Learning System, LLC, a subsidiary of MediMedia USA Inc., Third Edition 2003.

Tarnita D., Boborelu C., Popa D., Tarnita, C., Rusu, L., The three-dimensional modeling of the complex virtual human elbow joint, Romanian Journal of Morphology and Embryology, Vol 51, No.3, pp 489-495, 2010.

Teo Ee Chon, Zhang Qing Hang, Huang Russel, Finite element analysis of head-neck kinematics during motor vehicle accidents: Analysis in multiple planes, Medical Engineering \& Physics 29, Elsevier, pp. 54-60, 2007. 
Clinciu, M. \& Ciunel, S.: The Biofidelic Behaviour of a Cranio-Cervical Spine Syst...

Van der Horst Marike Joanne, Human Head Neck Response in Frontal, Lateral and Rear End Impact Loading - modelling and validation, $\mathrm{PhD}$ Thesis, Eindhoven University of Technology, Maastricht University and the TNO Crash Safety Centre Eindhoven: Technische Universiteit Eindhoven, Proefschrift. - ISBN 90-386-2843-9, 2002.

Yan J., A Computer Simulation Model Of The Human Head-Neck Musculoskeletal System, PhD Thesis, The University of Tennessee and The University of Memphis, December, 2006.

Adams, User Guide and Tutorials, 2001-2004

National Highway Traffic Safety Administration - "CRASH3 Technical Manual". http//www-nass. nhtsa. dot. gov/ NASS/MANUALS/Crash3Man.pdf

SolidWorks, User Guide and Tutorials, 1995-2010. 